\title{
Implementing TB Surveillance in Nigeria: Best Practices, Challenges and Lessons Learnt
}

\author{
Oluremilekun Comfort Kusimo*, Chukwuebuka Immanuel Ugwu, \\ Ufuoma Aduh, Chika Augustus Okoro
}

World Health Organization, Lagos, Nigeria

Email: *stremi2001@yahoo.com

How to cite this paper: Kusimo, O.C., Ugwu, C.I., Aduh, U. and Okoro, C.A. (2020) Implementing TB Surveillance in Nigeria: Best Practices, Challenges and Lessons Learnt. Journal of Tuberculosis Research, 8, 199-208.

https://doi.org/10.4236/jtr.2020.84018

Received: September 24, 2020

Accepted: November 7, 2020

Published: November 10, 2020

Copyright $\odot 2020$ by author(s) and Scientific Research Publishing Inc. This work is licensed under the Creative Commons Attribution International License (CC BY 4.0).

http://creativecommons.org/licenses/by/4.0/

\begin{abstract}
Introduction: TB Surveillance is a critical component of the global TB response. Comprehensive, accurate and timely information on TB is crucial for an effective $\mathrm{TB}$ control program hence the need for a robust tuberculosis surveillance system in all countries that contribute to the global burden of TB including Nigeria. Against this backdrop, an intervention was set in motion to triangulate the information from the health data reporting systems towards improving the overall surveillance system for TB in the country. Objectives: This article highlights the best practices, lessons learnt and challenges associated with the implementation of TB surveillance in Nigeria. In resource-limited settings such as Nigeria where health systems including health information management are sub-optimal, there is a heavy reliance on national and sub-national TB surveillance systems. TB data is mainly reported through the National Tuberculosis Control, however the integrated disease surveillance and response (IDSR) system also provides a platform for TB data collation through the LGA and State disease surveillance and notification officers. Conclusion: Implementing TB surveillance in Nigeria brought to fore the need for a wider engagement of all health facilities in TB control. As a dividend of the TB surveillance intervention, quality of care was improved in the private health sector through effective linkages to the commodity management system of the NTP and the national treatment guidelines. Strengthening community health surveillance system was identified as a critical element of Tuberculosis control. Also, the efficiency birthed by the integration of TB surveillance into the IDSR structure opened up other potential opportunities such as a unified capacity building of community informants on all notifiable diseases and the integration of reporting and risk communication for all health issues at the community level.
\end{abstract}

\section{Keywords}

Tuberculosis, Surveillance, IDSR, Health Information, TB Data 


\section{Background}

Public health surveillance is described as the ongoing systematic collection, collation, analysis and interpretation of health information for informed and prompt public health action [1] [2]. Surveillance is the heart and conscience of public health and provides the intelligence required to prevent, avert and manage epidemics [2]. Disease surveillance provides a bedrock for all other public health elements including health promotion, health financing and health policy development. Tuberculosis surveillance is the embodiment of all components of public health surveillance focused on TB data. It describes the monitoring of incident and prevalent cases; risk of infection and deaths as a result of Tuberculosis and the use of such information to control TB epidemics [3]. Demographic, geographic, diagnostics and behavioural data are all relevant in TB surveillance for both human and non-human primates [4] [5] [6]. Comprehensive, accurate and timely information is crucial for an effective $\mathrm{TB}$ control program hence the need for a robust TB surveillance system in all countries, including Nigeria, that contribute significantly to the global burden of TB [2] [7] [8].

TB surveillance affords countries and institutions the information required to develop effective strategies, targeted interventions and robust policies to address the scourge of the epidemics [4] [5] [9] [10]. Globally, TB surveillance has been recognized as a critical source of information on the burden and epidemiological pattern of TB [11] [12]. Complete, accurate and timely surveillance data is needed to monitor the quality of TB care [13]. This is even more pertinent in resource-limited settings such as Nigeria where health systems including health information management are sub-optimal and less advanced compared to industrialized countries [8] [14]. Robust health information systems are sufficiently able to assess the burden of TB based on case notification hence some countries can measure TB case incidence directly from their case notification [2]. This is not the case in countries with weak health information systems such as Nigeria. Annually, WHO has estimated the burden of TB in Nigeria and in 2012, a national prevalence survey was conducted to provide an empirical measurement of the incidence and prevalence of TB in the country. According to the 2019 global TB Report, the incidence of TB in Nigeria is 219 (143 - 311, 95\% CI) per 100,000 populations [15]. This incidence forms the basis for estimating the TB disease burden at national and sub-national levels with large margins of error due to a projected population estimate derived from a national census conducted far back in 2006.

\section{Introduction}

Tuberculosis is a disease of public health concern in Nigeria as it contributes significantly to the country's annual morbidity and mortality data [15]. Nigeria has the largest burden of TB in Africa and is among the ten countries accounting for $80 \%$ gap in the estimated incident cases and the number of incident cases reported globally [15]. Nigeria's treatment coverage for TB has remained pre- 
cariously low at $24 \%$ leaving a huge gap of undiagnosed TB in the population. Similarly, TB mortality was $64 / 100,000$ and $16 / 100,000$ for HIV negative and positive persons respectively [15]. In 2019, the country notified only 120,266 of the estimated 420,000 incident cases of TB, leaving a gap of 300,000 undiagnosed cases who remain infectious in the population with one case potentially infecting 10 to 15 others annually [15] [16]. Nigeria also contributes significantly to the global burden of Multi-Drug Resistant TB (MDR-TB). The World Health Organization estimates that the prevalence of Multi-Drug Resistant TB (MDR-TB) in Nigeria is 4.3 percent $(3.2-5.5)$ among new patients and 15 percent (11 - 19) among previously treated patients, for a total of $21,000(13,000-32,000)$ MDR-TB cases [15]. However, in 2019, only 2384 (11\% of estimated incident cases) persons with MDR-TB were notified, of whom 1975 (82\%) were enrolled into treatment. TB also has enormous negative economic impact on Nigerians with about $71 \%$ of the TB patients affected catastrophically as a result of the costs incurred in seeking care for the disease [15]. In spite of the huge public health issue presented by TB, the national surveillance system remains inadequate and unable to report every suspected and confirmed case of TB.

The Integrated Disease Surveillance and Response (IDSR) was created by WHO in 1988 to address weaknesses in health surveillance systems in the Africa region, however due to weak political will, this system remains poorly funded and unable to achieve its full potentials [17]. This setback in the IDSR has led to the creation of parallel data information systems at the national, state and LGA levels. Key among which is the National Health Management Information System (NHMIS). In country, WHO continues to support the IDSR system to notify epidemic prone diseases, diseases marked for elimination and eradication; and other diseases of public health concern, including TB. The system is however unequivocally focused on acute flaccid paralysis and to a lesser extent, cholera, measles and neonatal tetanus. This is a far cry from the original design of the IDSR which should report 42 priority diseases under surveillance in Nigeria with designated standard case definitions [18]. The flow of information through the IDSR is from prioritized health facilities (based on population and disease burden), where diseases that have epidemic potentials and those which are targeted for eradication and elimination, are reported either immediately or monthly to the surveillance focal persons in the health facility and then to the LGA through designated Disease surveillance and notification officer (DSNO) using appropriate IDSR reporting forms (001, 002 and 003). The LGA level data collated is then reported to the next level which is the State Ministry of Health $(\mathrm{SMoH})$.

At the community level, the IDSR structure relies on community informants to report notifiable diseases to the focal sites or directly to the LGA DSNO. The DSNO reports to the state DSNO and State Epidemiologist who then reports to the federal Ministry of Health via the Consultant Epidemiologist and the $\mathrm{Na}$ tional Centre for Disease Control (NCDC). 
Against this backdrop, an innovative strategy to strengthen TB surveillance system was deployed in 12 high burden states (Kaduna, Kano, Rivers, Delta, Lagos, Oyo, Niger, Benue, Imo, Anambra, Taraba and Bauchi) with USAID grant in 2018. The intervention was geared to support the IDSR system to improve TB notification in the 12 states.

\section{Principles of TB Surveillance in Nigeria}

The key construct of TB surveillance is to enable a robust and accurate assessment of the burden and epidemiological pattern of TB towards developing effective program designs and policies to address the TB epidemics. These epidemiological patterns describe the national and sub-national TB morbidity and mortality data; and the populations that are most affected by the disease. TB surveillance can therefore provide the critical information to guide specific TB preventive and control measures such as contact tracing, targeted TB screening and other interventions that can effectively control the transmission of $\mathrm{TB}$ in the general population. Ultimately, TB surveillance can generate information for a coordinated national and global TB response. Effective TB surveillance is underscored by a robust health information system that can facilitate a comprehensive, accurate and timely collation, collection and analysis of TB information at all levels. Hence, under-reporting and double counting are markers of a weak surveillance system and can impact negatively on programmatic responses for TB control [4].

\section{Scope of Implementation and Approach}

TB surveillance was strengthened in 12 states in Nigeria, namely Kaduna, Kano, Rivers, Delta, Lagos, Oyo, Niger, Benue, Imo, Anambra, Taraba and Bauchi, as shown in Figure 1 below. The intervention was supported by USAID and was focussed on three key activities: hospital based surveillance, community based surveillance, and health systems data strengthening. Underpinning this intervention was the theory that the "missing" TB cases could be in private hospitals operating outside the radar of the National TB Program (NTP), undiagnosed in the communities or diagnosed but not notified. In light of this, the hospital based surveillance was implemented mainly in private hospitals not previously linked to the NTP diagnostic, treatment, and M\&E systems. These facilities were referred to as non-NTP sites in the context of the TB surveillance intervention and were engaged through a coordinating umbrella body of the medical directors known as Association of General and Private Medical Practitioners of Nigeria (AGPMPN). Medical directors of the health facilities were sensitized on the current National TB management guidelines and linked to the TB commodity logistics systems including the specimen transportation networks. Standard operating procedures, job aids, and IEC materials were displayed in their waiting and consulting rooms.

Community based TB surveillance was conducted through targeted community 


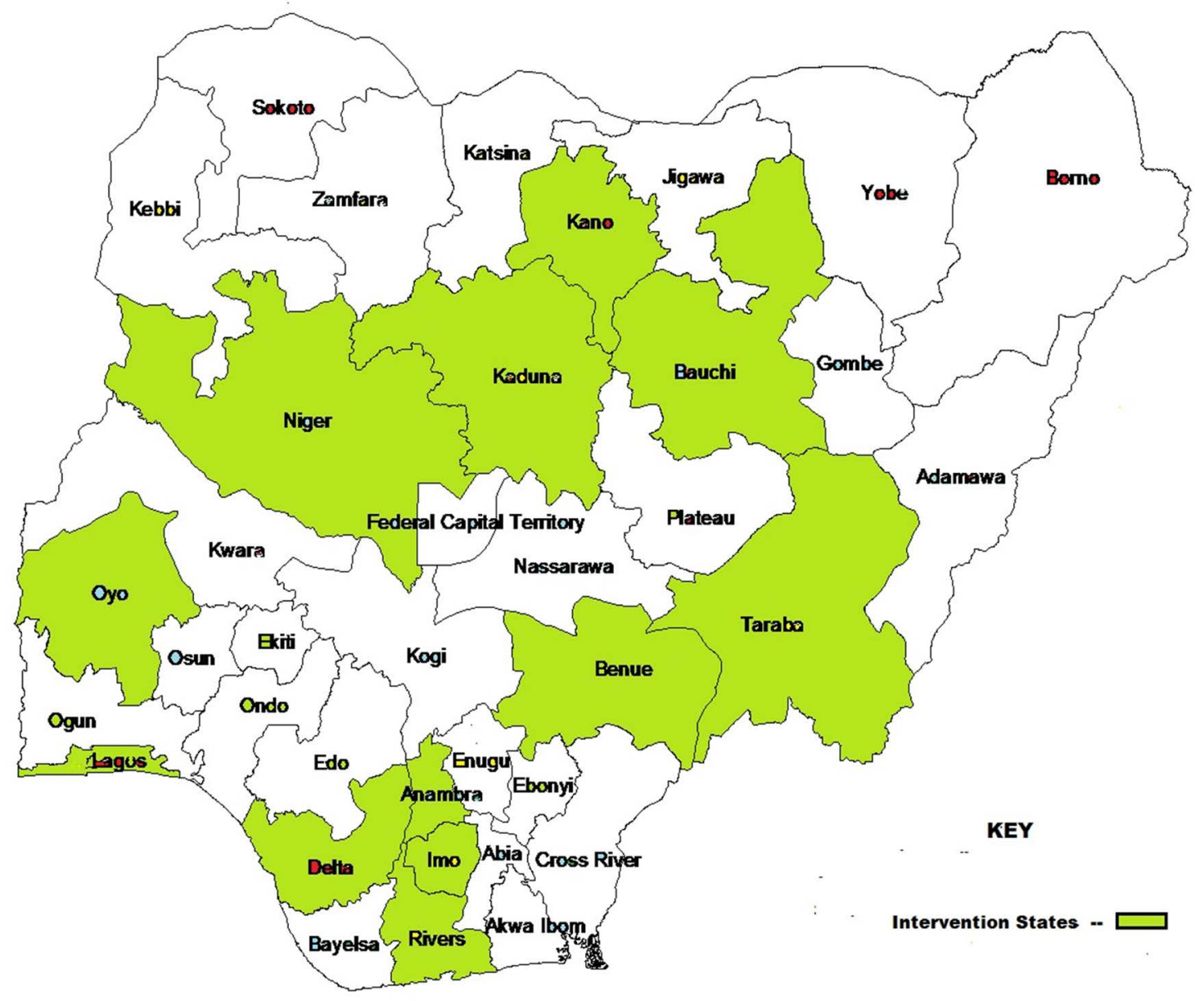

Figure 1. TB Surveillance in Nigeria-Intervention States.

outreaches, house-to-house surveys and the engagement of community informants in the identification and referral of presumptive TB clients. The outreaches and house to house surveys entailed collection of samples from symptomatic members of the community towards improving access to TB diagnostics and treatment services. Other elements of the community engagement include advocacy to community leaders and stakeholders including political and traditional leaders, religious leaders, men and women socio-cultural groups, heads of schools, and local traditional healthcare providers (bone-setters, birth attendants, etc.). This is followed by community sensitization using traditional means such as town announcers, announcement in places of worship and schools, as well as in group meetings and markets. Summarily, the TB surveillance intervention was hinged on the effective harmonisation of TB data generated from both the IDSR and the NTP M \& E systems. Although the IDSR structure covers a wider range of health facilities than the NTP, there were limitations with the quality of TB data generated through the structure. Hence, monthly meetings were conducted to harmonize data from the two systems while also leveraging on electronic reporting by the private health facilities who were non-NTP facilities. 


\section{Best Practices and Lessons Learnt}

Engagement of umbrella bodies of general and private medical practitioners served to rapidly expand the coverage of TB services in the private sector. Service expansion in this frequently ignored but thriving sector is critical and particularly so in settings where the vast majority of clients rely on their services [19]. A subtle and dynamic interplay of factors enhanced the engagement with the private sector which by nature thrives on profit generation. The facilities so engaged were linked to the NTP logistics system through which they had access to free anti-TB medicines for their patients. This improved quality of TB care in the engaged facilities by removing the need for the procurement of sub-standard, anti-TB medicines from unregulated markets [20]. Consequently, the cost of treating TB patients was reduced and this resulted in better patient adherence and treatment outcomes. Other incentives include the supply of reagents for AFB Microscopy.

Leveraging on technology enhanced the timeliness and usefulness of TB surveillance data and ensured prompt interventions for clients. Social media platforms such as WhatsApp were used to create groups for medical doctors in the private sector to enable real time notification of presumptive and diagnosed TB cases. This approach greatly shortened the time between diagnosis and treatment enrolment and minimised pre-treatment loss to follow up which was hitherto prevalent in the private sector [21]. Also, the platforms were used for communicating updates on $\mathrm{TB}$ treatment guidelines and for the provision of real-time clinical support for medical officers. The support resulted in an increase from 49,727 TB patients in 2018 to 54,979 TB patients in 2019 out of which the intervention accounted for 12\% (6657 TB patients) of TB cases notified in 2019. The TB surveillance has established mechanism for linking diagnosed TB cases from additional 3960 non-NTBLCP facilities to the M \& E system.

The targeted TB Surveillance interventions at the community level strengthened important linkages between PHCs and communities within their catchment areas. These community-facility linkages proved critical in improving overall surveillance across the various IDSR priority diseases. Using TB as the entry point, this intervention also increased the community uptake of other health services available at the PHCs.

Other components of the health systems such as health management information systems and human resource for health were also supported through the TB surveillance intervention. The data management systems of the IDSR and NTP M\&E systems at state levels benefited from the monthly data collation and harmonization meetings. Timeliness and completeness of data improved across the 12 states. Capacities on TB surveillance were built at facility, LGA and state levels. In addition, the intervention gave rise to monthly data collection and review of TB data in a program which hitherto reviewed data on a quarterly basis. This timely review of programmatic data resulted in prompt resolution of issues affecting program implementation at all levels. Beyond the monthly meetings, data 
harmonization with the TB program has now become an integral part of the IDSR system at the LGA and state level. This, undoubtedly, has improved the overall quality of TB data reported through the IDSR structure at all levels.

The surveillance intervention efficiently leveraged on the well-established Polio eradication initiative surveillance structure and processes. Structures such as annual immunization campaigns and community informants were utilised to cascade TB surveillance down to community level reaching all the hard to reach areas previously underserved by traditional TB services. Similarly, the efficiency birthed by the integration of TB surveillance into the IDSR structure opened up other potential opportunities such as a unified capacity building of community informants on all notifiable diseases, integrated reporting systems at community level, effective community awareness on all health issues that affect people across the life course, among others. Beyond that, it called attention to the urgent need for strengthening community health systems in a sustainable and cost-efficient manner.

\section{Challenges with TB Surveillance in Nigeria}

Sub-optimal TB notification has remained an issue for the TB Program in Nigeria. This challenge can be attributed to weak policy framework on mandatory notification of TB by all health facilities and the outcome is under-reporting of TB cases especially in facilities that are not engaged by the National TB program as TB treatment centres. Considering that over $80 \%$ of health facilities are yet to be included in the data management system of the NTP, this presents a major challenge for TB surveillance. This is further exacerbated by a weak community surveillance system which means that persons who are unable to access health services are also missed in the community. These challenges are not unrelated to the poorly funded IDSR system, low awareness about TB in the general population and the failure to achieve universal health coverage in Nigeria.

Poorly implemented regulatory framework results in multiplicity of data management systems which is chaotic and counterproductive. An instance is the multiple electronic TB data capture systems being deployed at both community and health facility levels by different implementing partners. Each unique platform addresses the peculiar need of specific funders. This creates duplicity of functions and can significantly impinge on the time available to LGA staff to carry out other important responsibilities.

Nationally, there is little integration between the Epidemiology Unit of the FMoH (now metamorphosed into the National Centre for Disease Control) and various disease control programs. The impact of this non-integration is parallelism at the lower levels. The case definition for Tuberculosis in the IDSR guidelines differs markedly from the case definition of TB being used by the NTP as recommended by WHO thereby leading to misclassification and under-reporting of TB cases on the IDSR structure. Also, a key drawback is the paucity of adequate human resource and technical capacities at the lower rungs of the health 
system in various parts of the country. There is evidence to suggest that poor understanding of the reporting requirements of the IDSR structure among health care workers has impacted negatively on the quality of public health surveillance data in the country [18] [22] [23]. Many healthcare workers, including physicians, do not have adequate knowledge of the persons or authorities to whom they are expected to report notifiable diseases; while those who have knowledge are hindered by lack of infrastructure and adequate logistics [22].

Clinicians have a significant role in disease surveillance and reporting given that they have the ability to cascade knowledge down to other cadres of the health care system and therefore should have their capacity properly built on the use of the IDSR structure [18]. Many healthcare workers have adequate knowledge of the use of IDSR003 form but have very limited knowledge of the use of other very critical IDSR forms [23]. Lack of robust training on disease surveillance affects the effectiveness of the surveillance system; this is also reflected in the absence of feedback to healthcare workers in peripheral facilities [24].

\section{Implication for Future Studies}

Future studies should explore the empirical measurement of the impact of TB surveillance on TB control in Nigeria. Investigating the relevance of non-traditional surveillance data such as pharmacy and patent medicine vendors' dispensing information to TB surveillance in Nigeria may also provide useful insights.

\section{Conflicts of Interest}

The authors declare no conflicts of interest regarding the publication of this paper.

\section{References}

[1] Castro, K.G. and Castro, K.G. (2007) Tuberculosis Surveillance: Data for Decision-Making. Clinical Infectious Diseases, 44, 1268-1270.

[2] Falzon, D. and A1, F. (2007) What Is Tuberculosis Surveillance in the European Union Telling Us? Clinical Infectious Diseases, 44, 1999-2003. https://doi.org/10.1086/514343

[3] Washko, R.M. and Frieden, T.R. (1996) Tuberculosis Surveillance Using Death Certificate Data, New York City, 1992. Public Health Reports, 111, 251-255.

[4] Rieder, H.L., Watson, J.M., Raviglione, M.C. and Forssbohm, M. (1996) Surveillance of Tuberculosis in Europe. European Respiratory Journal, 9, 1097-1104. https://doi.org/10.1183/09031936.96.09051097

[5] Ghosh, S., Moonan, P.K., Cowan, L., Grant, J., Kammerer, S. and Navin, T.R. (2012) Infection, Genetics and Evolution Tuberculosis Genotyping Information Management System: Enhancing Tuberculosis Surveillance in the United States. Infection, Genetics and Evolution, 12, 782-788. https://doi.org/10.1016/j.meegid.2011.10.013

[6] Lerche, N.W., Yee, J.L., Capuano, S.V. and Flynn, J.L. (2008) New Approaches to Tuberculosis Surveillance in Nonhuman Primates. ILAR Journal, 49, 170-178.

[7] Rafei, A., Pasha, E. and Orak, R.J. (2012) Tuberculosis Surveillance Using a Hidden Markov Model. Iranian Journal of Public Health, 41, 87-96. 
[8] Dairo, M.D., Bamidele, J.O. and Adebimpe, W.O. (2010) Disease Surveillance and Reporting in Two Southwestern States in Nigeria: Logistic Challenges and Prospects. Journal of Public Health and Epidemiology, 2, 125-129.

[9] Yokoe, D.S., Subramanyan, G.S., Nardell, E., Sharnprapai, S., Mccray, E. and Platt, R. (1999) Supplementing Tuberculosis Surveillance with Automated Data from Health Maintenance Organizations. Emerging Infectious Diseases, 5, 779-787. https://doi.org/10.3201/eid0506.990606

[10] Sprinson, J.E., Lawton, E.S., Porco, T.C., Flood, J.M. and Westenhouse, J.L. (2006) Assessing the Validity of Tuberculosis Surveillance Data in California. BMC Public Health, 6, Article No. 217. https://doi.org/10.1186/1471-2458-6-217

[11] Fiebig, L., Kollan, C., Hauer, B., Gunsenheimer-Bartmeyer, B. and Der, M. (2012) HIV-Prevalence in Tuberculosis Patients in Germany, 2002-2009: An Estimation Based on HIV and Tuberculosis Surveillance Data. PLOS ONE, 7, 2002-2009. https://doi.org/10.1371/journal.pone.0049111

[12] García-basteiro, A.L., Schaaf, H.S., Diel, R. and Migliori, G.B. (2018) Adolescents and Young Adults: A Neglected Population Group for Tuberculosis Surveillance. European Respiratory Journal, 51, Article ID: 1800176. https://doi.org/10.1183/13993003.00176-2018

[13] Ditah, I.C., et al. (2008) Monitoring Tuberculosis Treatment Outcome: Analysis of National Surveillance Data from a Clinical Perspective. Thorax, 63, 440-446. https://doi.org/10.1136/thx.2006.073916

[14] Mor, Z., Migliori, G.B., Althomsons, S.P., Loddenkemper, R., Trnka, L. and Iademarco, M.F. (2008) Comparison of Tuberculosis Surveillance Systems in Low-Incidence Industrialised Countries. European Respiratory Journal, 32, 1616-1624. https://doi.org/10.1183/09031936.00042908

[15] WHO (2019) Global TB Report.

[16] Raviglione, M. and Sulis, G. (2016) Tuberculosis 2015: Burden, Challenges and Strategy for Control and Elimination. Infectious Disease Reports, 8, 33-37. https://doi.org/10.4081/idr.2016.6570

[17] Disease, I. (2005) National Policy (IDSR) Federal Ministry of Health.

[18] Isere, E.E., Fatiregun, A.A. and Ajayi, I.O. (2015) An Overview of Disease Surveillance and Notification System in Nigeria and the Roles of Clinicians in Disease Outbreak Prevention and Control. Nigerian Medical Journal, 56, 161-168. https://doi.org/10.4103/0300-1652.160347

[19] Lei, X., Liu, Q., Escobar, E., Philogene, J., Zhu, H. and Wang, Y. (2015) Public-Private Mix for Tuberculosis Care and Control: A Systematic Review. International Journal of Infectious Diseases, 34, 20-32. https://doi.org/10.1016/j.ijid.2015.02.015

[20] Onwujekwe, O., et al. (2009) Quality of Anti-Malarial Drugs Provided by Public and Private Healthcare Providers in South-East Nigeria. Malaria Journal, 8, Article No. 22. https://doi.org/10.1186/1475-2875-8-22

[21] Ukwaja, K.N., Alobu, I., Nweke, C.O. and Onyenwe, E.C. (2013) Healthcare-Seeking Behavior, Treatment Delays and Its Determinants among Pulmonary Tuberculosis Patients in Rural Nigeria: A Cross-Sectional Study. BMC Health Services Research, 13, 25. https://doi.org/10.1186/1472-6963-13-25

[22] Lafond, K.E., et al. (2014) Notifiable Disease Reporting among Public Sector Physicians in Nigeria: A Cross-Sectional Survey to Evaluate Possible Barriers and Identify Best Sources of Information. BMC Health Services Research, 14, 568. https://doi.org/10.1186/s12913-014-0568-3 
[23] Jinadu, E., Adebiyi, K.A., Sekoni, A.O. and Bambgoye, O.O. (2018) Integrated Disease Surveillance and Response Strategy for Epidemic Prone Diseases at the Primary Health Care (PHC) Level in Oyo State, Nigeria: What Do Health Care Workers Know and Feel? The Pan African Medical Journal, 31, 19.

https://doi.org/10.11604/pamj.2018.31.19.15828

[24] Lar, L.A., Afolaranmi, T.O., Tagurum, Y.O., Uzochukwu, B. and Zoakah, A.I. (2015) Challenges of Integrated Disease Surveillance Response Reporting among Healthcare Personnel in Mangu, Plateau State, Nigeria. Journal of Public Health and Epidemiology, 7, 108-113. https://doi.org/10.5897/JPHE2015.0714 